\title{
NaI AND CaII INTERSTELLAR ABSORPTION IN THE FIELD OF SN 1987A
}

\author{
P. MOLARO ${ }^{1}$, G. VLADILO ${ }^{1}$, S. D'ODORICO ${ }^{2}$, M. DENNEFELD $^{3}$, R. FERLET $^{3}$, \\ A. VIDAL-MADJAR ${ }^{3}$ \\ 1 Trieste Astronomical Observatory 2 European Southern Observatory \\ 3 Institute d'Atrophysique de Paris
}

\begin{abstract}
We present CaII and NaI spectra for a sample of stars in a 30'x30' field connecting 30 Dor with SN 1987A. Observations were taken with the CES spectrograph linked via fibres with the $3.6 \mathrm{~m}$ ESO telescope at a resolution of $\sim 6 \mathrm{~km} \mathrm{~s}^{-1}$. The LMC gas shows a complex structure with both single and multiple components spanning 250 to $300 \mathrm{~km} \mathrm{~s}^{-1}$. Results can be interpreted using the multi-layer structure suggested by $21 \mathrm{~cm}$ observations. Comparing our optical absorptions with $21 \mathrm{~cm}$ emissions yields evidence that layers with increasing radial velocity are located one behind the other. The intermediate velocity components are numerous in CaII, but rare in NaI. Towards Sk-69 211, for which we have the highest S/N Call spectrum, we have detected a number of components comparable to that found in SN 1987A. The $64 \mathrm{~km} \mathrm{~s}^{-1}$ cloud is detected in a well-confined region of the field, showing remarkable constancy in the radial velocity, which we interpret as evidence that this cloud is located close to the Galaxy.
\end{abstract}

\section{Introduction}

We present here the first results of the interstellar sub-group of the ESO key-programme on "Coordinated Investigations of Selected Regions in the Magellanic Clouds" which started last winter. The basic aims of the key-programme have been outlined by de Boer et al. (1989) and those concerning the interstellar investigations, by Ferlet and Vidal-Madjar (1989). As a first step in our observing program, we investigated the Selected Region around SN 1987A. The NaI and CaII observations were made in November 1989 at ESO, La Silla, using the Coudé Echelle Spectrograph (CES) fed with a $40 \mathrm{~m}$-long optical fibre from the Cassegrain focus of the $3.6 \mathrm{~m}$ telescope.

\section{LMC components}

Absorption components with $V_{H E L} \geq 200 \mathrm{~km} \mathrm{~s}^{-1}$ probably originate in the main body of the Large Magellanic Cloud (LMC). The interstellar gas at these velocities shows a complex structure with both single and multiple components, with differing radial velocities and strengths. In some cases at least three absorption components are detected, spanning 250 to $300 \mathrm{~km} \mathrm{~s}^{-1}$. Results can be interpreted using the multi-layer structure suggested by $21 \mathrm{~cm}$ observations. The data are in general consistent with the existence of extended layers or neutral gas. Two NaI absorptions at $V_{H E L} \simeq 270$ - $280 \mathrm{~km} \mathrm{~s}^{-1}$ and at $V_{H E L} \simeq 290 \mathrm{~km} \mathrm{~s}^{-1}$ corresponding to the two strongest HI emission components detected by Rohlfs et al. (1984) in our field are observed. Often the NaI absorption breaks down into a more complex structure, with faint resolved components or asymmetries. In the direction of Sk-69 239, which is angularly close to the 30-Dor complex, a red absorption wing, observed in CaII but not in NaI, extends from about 200 to $270 \mathrm{~km} \mathrm{~s}^{-1}$. The stars where the $V_{H E L} \simeq 290 \mathrm{~km} \mathrm{~s}^{-1}$ absorption is present have the richest interstellar spectra. This, together with the 
lack of detection of the $290 \mathrm{~km} \mathrm{~s}^{-1}$ absorption in some lines of sight where it is visible in emission, suggests that the $V_{H E L} \sim 290 \mathrm{~km} \mathrm{~s}^{-1}$ component is located beyond the others. In general, our data are consistent with the location of the layers at higher and higher velocities one beyond the other. Another, rather strong, absorption is present at $V_{H E L} \simeq 260 \mathrm{~km} \mathrm{~s}^{-1}$. The component is pervasive in the Eastern region of our field with a possible steep edge in the N-S direction, approximately crossing the SN 1987A. This component is the only interstellar absorption in Sk-69 216 observable at LMC velocities, suggesting that this layer too, is closer to us than the others at higher velocities.

\section{Intermediate velocity components}

The most prominent feature at intermediate velocities is the $64 \mathrm{~km} \mathrm{~s}^{-1}$ cloud. Towards Sk-69 211 the CaII absorption for this component is even stronger than the Galactic and LMC interstellar absorptions. This feature is observed in a well-localized region of our field. It is observed towards Sk-69 211, Sk-69 216, SN 1987A, R127 and R128 but not towards Sk-69 195 and Sk-69 204, vignetting an elongated feature of at least 15 arcmin in the $\mathrm{N}-\mathrm{S}$ direction. The measured radial velocity shows an impressive constancy, with variations of less than $1 \mathrm{~km} \mathrm{~s}^{-1}$ among the different lines of sight, which is of the order of the wavelength accuracy. Such a situation is not encountered in the other LMC absorption features. Fifteen arcmin correspond to about $220 \mathrm{pc}$ at the distance of the LMC and it seems implausible to have the radial velocity constant over such a large scale. We interpret this as evidence that this cloud is not located in the LMC but is much closer to the Galaxy, probably in the Galactic halo. Further evidence is provided by the detection of the cloud towards LMC stars located in the foreground with respect to the main body of the LMC, as pointed out by Molaro et al. (1989).

At other velocities the CaII $\mathrm{K}$ spectra appear rather rich in weak components while the $\mathrm{NaI}$ spectra are free of absorptions greater than $\approx 4 \mathrm{~mA}$. In particular, towards Sk-69 211, for which we have the spectrum with the highest signal-to-noise, we have been able to detect at least four other additional components at $90 \mathrm{~km} \mathrm{~s}^{-1}, 107 \mathrm{~km} \mathrm{~s}^{-1}, 128 \mathrm{~km} \mathrm{~s}^{-1}$ and $170 \mathrm{~km} \mathrm{~s}^{-1}$. We argue that such a degree of complexity may be the rule for the other lines of sight of the MCs when observed with comparable S/N. These components have no counterparts observable in NaI and in the most stringent case the $\mathrm{EW}(\mathrm{CaII}) / \mathrm{EW}(\mathrm{NaI})$ ratio is greater than 10 , showing evidence of the RoutlySpitzer effect. Thus, CaII appears to be a better tracer than NaI for the detection of high-velocity material. Despite the fact that Sk-69 211 is located at only 7 arcmin from SN 1987A, these absorptions have different velocities with respect to the SN 1987A components $(76,121,125$ $\mathrm{km} \mathrm{s}^{-1}$ ). The $90 \mathrm{~km} \mathrm{~s}^{-1}$ and the $170 \mathrm{~km} \mathrm{~s}^{-1}$ components are also seen toward Sk-69 216, which is located at 2.2 arcmin apart, suggesting a smaller scale for the size of these clouds.

\section{References}

de Boer, K.S., Azzopardi, M. ,Baschek, B., Dennefeld, M., Israel, F.P., Molaro, P., Seggewiss, W., Spite, F., Westerlund, B.E. (1989), The Messenger 57, 27.

Ferlet, R., Vidal-Madjar, A. (1989), Recent Developments of Magellanic Cloud Research, K.S. de Boer, F. Spite, G. Stasinka (eds.) (Observatoire de Paris) p.99.

Molaro, P., Vladilo, G., Avila, G., D'Odorico, S. (1989), Astrophys. J. 339, L63.

Rohlfs, K., Kreitschmann, J., Siegman, B.C., Feitzinger, J.V. (1984), Astron. Astrophys. 137, 343. 